Series A

I. MATHEMATICA

492

\title{
NOTE ON THE DISTRIBUTION OF IRREGULAR PRIMES
}

BY

TAUNO METSÄNKYLÄ

H E L I N K I 1971

S U O M A L A I N E T I E D A K A T EM I A

doi:10.5186/aasfm.1971.492 
Copyright (C) 1971 by

Academia Scientiarum Fennica

Communicated 15 January 1971 by K. A. INkERI 


\section{Note on the distribution of irregular primes}

1. Introduction. A prime $p$ is said to be irregular if it divides the numerator of at least one of the Bernoulli numbers $B_{2}, B_{4}, \ldots, B_{p-3}$ (in the even suffix notation). The simplest proof for the known fact that the number of irregular primes is infinite was given by CARLITZ [1]. JENSEN [2] proved the stronger result that there is an infinity of irregular primes $\equiv-1(\bmod 4)$, and Montgomery [3] generalized this as follows: for every integer $T>2$, there are infinitely many irregular primes $\neq 1$ $(\bmod T)$. This result also contains the proposition asserted by SLAvuTSkII [5], namely, that the number of irregular primes $\equiv-1(\bmod 3)$ is infinite.

SlavutskiI remarked that some of the known irregular primes $\equiv-1$ $(\bmod 3)$ are $\equiv 1(\bmod 4)$. According to Montgomery, the first 216 irregular primes, grouped modulo 12, split into groups of $49,66,43$, and 58 primes. More generally, as noted in [3], numerical results indicate that there is no deficiency of irregular primes in the residue class $1(\bmod T)$, if $T>2$.

In this note we shall show that there are infinitely many irregular primes $\equiv \pm 5(\bmod 12)$, so that the following theorem holds true:

Theorem 1. At least one of the residue classes $1(\bmod 3)$ and $1(\bmod 4)$ contains an infinite number of irregular primes.

In addition, using ideas from [3], we shall generalize this result by proving

Theorem 2. For every integer $T>4, T \neq 6$, there are infinitely many irregular primes $\neq \pm 1(\bmod T)$.

We also wish to mention the connexion between the questions about the distribution of irregular primes and the number of regular primes. This number has been conjectured to be infinite ([4], cf. also [7]). The conjecture is proved if, for some integer $T$, there exists a residue class (mod $T$ ) prime to $T$ containing only a finite number of irregular primes. However, in view of our present knowledge about irregular primes, the existence of such a residue class seems improbable. 
2. Preliminary results. Write the Bernoulli numbers in the form

$$
B_{2 k}=N_{2 k} / D_{2 k}
$$

(in lowest terms) with $D_{2 k}>0$. Then, by the known Staudt-Clausen theorem, $D_{2 k}$ is the product of those distinct primes $l$ for which $l-1$ divides $2 k$. Furthermore, by setting

$$
S_{2 k}(t)=1^{2 k}+2^{2 k}+\ldots+(t-1)^{2 k}
$$

we can state that $N_{2 k}$ is comnected with $D_{2 k}$ by the congruences

$$
t N_{2 k} \equiv D_{2 k} S_{2 k}(t)\left(\bmod t^{2}\right),
$$

valid for each positive integer $t[6, \mathrm{p} .260]$.

Those prime divisors of $N_{2 k}$ which divide the numerator of $N_{2 k} / k$ are called proper. As is known (see, e.g., [3]), every prime which is a proper divisor of some $N_{2 k}$ is irregular.

To be able to use (1), we shall need some information about $S_{2 k}(t)$. If $P$ denotes an arbitrary odd prime, we have $[3, \mathrm{p} .555]$

$$
S_{2 k}(P) \equiv P / 6\left(\bmod P^{2}\right) \text { for } k \equiv 1(\bmod P(P-1)) .
$$

Moreover, assuming that $k>1$ the following congruences can be easily established:

$$
\begin{aligned}
S_{2 k}(8) & \equiv-12(\bmod 32) \text { for } k \equiv 1(\bmod 4) \\
S_{2 k}(9) & \equiv-3(\bmod 27) \text { for } k \equiv 1(\bmod 9) \\
S_{2 k}(12) & \equiv-10(\bmod 24)
\end{aligned}
$$

3. Proof of theorem 1. Let us suppose that there exists only a finite set of irregular primes $\equiv \pm 5(\bmod 12)$, say $, p_{1}, \ldots, p_{s}$. Put

$$
A=\left(p_{1}-1\right) \ldots\left(p_{s}-1\right)
$$

and consider $B_{2 q}$ with a prime $q \equiv 1(\bmod 12 A)$.

It is seen that $D_{2 q}=6$. Hence, by (1),

$$
12 N_{2 q} \equiv 6 S_{2 q}(12)\left(\bmod 12^{2}\right) \text {, }
$$

which combined with (5) yields

$$
N_{2 q} \equiv-5(\bmod 12) .
$$

From this congruence it follows that $N_{2 q}$ must contain a prime factor $p \neq \pm 1(\bmod 12)$. Since $p \neq q$, we conclude that $p$ is a proper divisor of $N_{2 q}$ and thus irregular. By our assumption, $p$ then appears in the above set of primes. 
Now, because $N_{2 q}$ contains a prime $p_{i}(1 \leqq i \leqq s)$ as a factor, the congruence

$$
B_{2 q} / q \equiv 0\left(\bmod p_{i}\right)
$$

holds true. On the other hand, by virtue of $q \equiv 1\left(\bmod p_{i}-1\right)$, the socalled Kummer's congruence gives us

$$
B_{2 q} / q \equiv B_{2} / 1=1 / 6\left(\bmod p_{i}\right),
$$

and we have a contradiction.

4. Proof of theorem 2. It is sufficient to prove, that the number of irregular primes $\neq \pm 1(\bmod t)$ is infinite for $t=8,9,12$, and $P$ (an arbitrary prime $>3$ ). Indeed, every integer $T>4, T \neq 6$, is divisible by at least one of these numbers $t$.

For $t=12$, the proof was carried out above. The case $t=9$ can be treated analogously by choosing $q \equiv 1(\bmod 18 A)$, whereupon $(6)$ is replaced by

$$
9 N_{2 q} \equiv 6 S_{2 q}(9)\left(\bmod 9^{2}\right)
$$

which gives, by (4), the congruence

$$
N_{2 q} \equiv-2(\bmod 9) \text {. }
$$

The remaining cases are more complicated. In the first place, let $P$ be a prime $>3$ and suppose, contrary to our assertion, that $p_{1}, \ldots, p_{s}$ are the irregular primes $\neq \pm 1(\bmod P)$.

We put

$$
M=6 P(P-1)\left(p_{1}-1\right) \ldots\left(p_{s}-1\right)=P^{h} M_{1},
$$

where $M_{1}$ is not divisible by $P$, and choose a prime $l$ satisfying

$$
l \equiv-1\left(\bmod 2 M_{1}\right), \quad l \equiv 3\left(\bmod P^{h}\right) .
$$

Then $l \neq \pm 1(\bmod P)$, and we can find a factor $n$ of $\frac{1}{2}(l-1)$ such that $D_{2 n}$, the denominator of $B_{2 n}$, is of the form $6 a l^{\prime}$ where $a \equiv \pm 1$ $(\bmod P)$ and $l^{\prime}(=2 n+1)$ is a prime $\neq \pm 1(\bmod P)$. (See [3], proof of theorem 3.1, where $n$ is denoted by $\mu^{\prime}$.)

Note that $l$ is chosen such that $\left(\frac{1}{2}(l-1), M\right)=1$. Consequently, $\left(n, l^{\prime} M\right)=1$ and the congruence

$$
n q \equiv 1\left(\bmod l^{\prime} M\right)
$$

is solvable for $q$. Moreover, one can assume $q$ to be a prime satisfying simultaneously with (9) also 


$$
2 d_{j} q \equiv-1\left(\bmod l_{j}^{2}\right) \quad(i=1, \ldots, r),
$$

where $d_{1}, \ldots, d_{r}$ are the divisors of $n$ and $l_{1}, \ldots, l_{r}$ are distinct primes $>l^{\prime} M$.

Consider $B_{2 Q}$ with $Q=n q \equiv 1\left(\bmod l^{\prime} M\right)$. Then $(10)$ assures us that $D_{2 Q}$ has no other prime factors than those of $D_{2 n}$, that is,

$$
D_{2 Q}=D_{2 n}=6 a l^{\prime}, \quad a \equiv \pm 1(\bmod P) .
$$

Applying this with (2) to the congruence

$$
P N_{2 Q} \equiv D_{2 Q} S_{2 Q}(P)\left(\bmod P^{2}\right)
$$

we get

$$
N_{2 Q} \equiv \pm l^{\prime}(\bmod P) .
$$

To eliminate the improper divisors of $N_{2 Q}$, we must write $Q=Q_{1} Q_{2}$ with $\left(Q_{1}, Q_{2}\right)=1$ and $Q_{2}$ containing exactly those primes of $Q$ that divide $D_{2 Q}$. Then $Q_{1}$ divides $N_{2 Q}$ (see, e.g., [6, p. 261]) and thus the numerator of $N_{2 Q} / Q$ equals $N_{2 Q} / Q_{1}$. Now, because $Q \equiv 1\left(\bmod 6 l^{\prime}\right)$, none of the prime factors 2,3 , and $l^{\prime}$ of $D_{2 Q}$ appears in $Q_{2}$ so that, by $(11), Q_{2} \equiv \pm 1(\bmod P)$, and we have $Q_{1} \equiv \pm Q \equiv \pm 1(\bmod P)$. Together with (13) this yields

$$
N_{2 Q} / Q_{1} \equiv \pm l^{\prime}(\bmod P) .
$$

Hence $N_{2 Q}$ contains a proper prime factor $\neq \equiv \pm 1(\bmod P)$ and the proof can be finished similarly as in the above cases.

As for the case $t=8$, one has to modify slightly the preceding proof. In fact, the formulas (7), (8), and (12) are replaced by

$$
\begin{gathered}
M=24\left(p_{1}-1\right) \ldots\left(p_{s}-1\right)=2^{h} M_{1} \quad\left(M_{1} \text { odd }\right), \\
l \equiv-1\left(\bmod M_{1}\right), \quad l \equiv 3\left(\bmod 2^{h}\right), \\
8 N_{2 Q} \equiv D_{2 Q} S_{2 Q}(8) \\
\left(\bmod 8^{2}\right),
\end{gathered}
$$

the last of which then gives, by (3), the crucial congruence

$$
N_{2 Q} \equiv \pm l^{\prime}(\bmod 8) \text {. }
$$

University of Jyväskylä

and

University of Turku

Finland 


\section{References}

[1] Carlitz, L.: Note on irregular primes. - Proc. Amer. Math. Soc. 5 (1954), 329-331.

[2] Jensen, K. L.: Om talteoretiske Egenskaber ved de Bernoulliske Tal. - Nyt Tidsskrift for Matematik 26, Afd. B (1915), 73-83.

[3] Montgomery, H. L.: Distribution of irregular primes. - Illinois J. of Math. 9 (1965), $553-558$.

[4] Siegel, C. L.: Zu zwei Bemerkungen Kummers. - Nachr. Akad. Wiss. Göttingen Math.-Phys. Kl. II Nr. 6 (1964), 51-57.

[5] Slavutskiř, I. S̆. [И. ІІ. С.тавутский]: К вопросу о простых иррегулярных числах. - Acta Arith. 8 (1963), 123-125.

[6] Uspensky, J. V., and Heaslet, M. A.: Elementary number theory. New York (1939).

[7] VANDiver, H. S.: Is there an infinity of regular primes? - Scripta Math. 21 (1955), $306-309$. 